\title{
A MATHEMATICAL MODEL USING AHP PRIORITIES FOR SOCCER PLAYER SELECTION: A CASE STUDY
}

\author{
E. Ozceylan
}

\section{ARTICLE INFO}

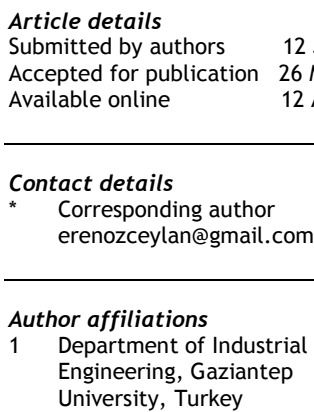

http://dx.doi.org/10.7166/27-2-1265
The process of player selection in multi-player sports like soccer is a complex multi-criteria problem. In this paper, a two-phase approach is proposed for soccer player selection. In the first phase, the attributes of each player - based on their position within a soccer team - is prioritised using the Analytic Hierarchic Process (AHP). In the second phase, a $0-1$ integer linear programming model is developed using the weights of player attributes, and the top performers are determined for inclusion in the team. Finally, a case study on the Turkish soccer club called Fenerbahçe is used to illustrate the applicability and performance of the proposed approach.

\section{OPSOMMING}

Die proses van speler-seleksie in spansporte soos sokker is ' $\mathrm{n}$ ingewikkelde, multikriteria probleem. ' $n$ Twee-fase benadering om die kies van spelers vir 'n sokkerspan word voorgestel. In die eerste fase word die eienskappe van die speler prioritiseer deur 'n analitiese hiërargie proses. Die prioritisering is gebaseer op hulle posisie in die span. In die tweede fase word 'n binêre lineêre programmeringsmodel ontwikkel deur gewigte aan 'n speler se eienskappe toe te ken. Toppresteerders word so identifiseer en in die span ingesluit. Laastens word 'n gevallestudie op die Turkse klubspan Fenerbahçe gebruik om die toepaslikheid en vertoning van die voorgestelde benadering te bepaal.

\section{INTRODUCTION}

In any sport, formation of a good team is vital to its success. Player selection for a team in most sports is a subjective issue, commonly based on the coaches' notions of what is needed to form the best team. Poor team selection lead to failure, costing a soccer team a trophy or a championship, not to mention the money; and the selection committee and the sport's organising authorities will be accountable to the people who love sport. Poor team selection can also affect the loyalty of players due to non-optimal and combinations of players.

Selection of players in a team is always a difficult task because a large number of qualitative and quantitative attributes in the player selection process need to be taken into account [1]. Thus a systematic approach such as mathematical modelling or the multi-criteria decision-making (MCDM) approach is essential. Although team management uses a variety of assessments such as testing, practice matches, and coaches' judgments to select and evaluate players, quantitative approaches could greatly benefit the team management and be significantly useful [2]. To avoid the traditionally vague and subjective practice of team selection, quantitative approaches could provide more objective results in a shorter time-frame.

In this paper, a two-phase approach is applied to select the best soccer players from within a particular team. In the first phase, the criteria of each player based on their position within the team are prioritised using AHP. In the second phase, a 0-1 integer linear programming model is 
developed, and the top performers are determined using the weights of the player criteria. To the best knowledge of the authors, this is the first study that combines AHP and mathematical modelling approaches to evaluate and select soccer players. The AHP methodology, integrated with the integer programming model, has been adopted as an alternative to the conventional and singular methods of weight derivation in AHP.

The remainder of this paper is organised as follows. In Section 2, a literature review is presented. In Section 3, an overview of the evaluation criteria is provided. In Section 4, attribute weights are estimated by AHP, and the details of the proposed approach are presented. In Section 5, the mathematical model for soccer player selection is presented. In Section 6, a case study to illustrate the effectiveness and applicability of the proposed approach is presented. Finally, in Section 7, the conclusions and future research directions are provided.

\section{LITERATURE REVIEW}

Many researchers have applied various models to team selection, such as the genetic algorithm [2,3], mathematical programming [4,5], ordered weighted averaging [6], AHP [7], and a fuzzy inference system [1]. Omkar and Verma [8] developed a solution approach based on the genetic algortihm to select players for a cricket team. A linear optimisation model for the optimal line-up of a volleyball team was developed by Boon and Sierksma [4]. Their proposed model was used to help coaches determine the optimal line-up of teams and to help scouting crews to assess and hire new players [4]. However, evaluation criteria were ignored in their model [4]. Merigo and Gil-Lafuente [6] used an ordered weighted averaging operator to select a football player from alternatives for a team in a transfer season. To do so, they considered the use of the Hamming distance, the adequacy coefficient, and the index of maximum and minimum level [6].

Gerber and Sharp [9] describe the ability-indexing and integer programme used, and discuss the results of an empirical study conducted using the statistics of 32 South African cricket players. Sharp et al. [5] develop an integer programming approach to determine the optimal cricket team by quantifying cricket players' performance based on their ability. Kamble et al. [7] present a procedure for selecting cricket team players in complex situations using AHP. Tavana et al. [1] propose a two-phase framework for player selection and team formation in soccer. The first phase in their study evaluates the alternative players with a fuzzy ranking method, and selects the top performers for inclusion in the team [1]. The second phase evaluates the alternative combinations of the selected players with a fuzzy inference system, and selects the best combinations for team formation [1]. Ahmed et al. [3] apply multi-objective optimisation and decision-making approaches to cricket team selection. They propose a novel gene representation scheme and a multi-objective approach that uses the genetic algorithm to optimise the overall batting and bowling strength of a team with 11 players as variables [3]

Amin and Sharma [10] suggest a new method for cricket team selection that uses the data envelopment analysis (DEA). They propose a DEA formulation for the evaluation of cricket players with different capabilities using multiple outputs [10]. This evaluation differentiates the efficient and inefficient cricket players, and ranks them on the basis of DEA scores [10]. Sarda et al. [2] present a solution approach based on the genetic algorithm to find the optimal solution for the problem of football team selection and formation. Their proposed model combines the generally used quantitative approach with attributes based on personal performance, team performance, and the collaborative performance of a player in the presence of other players in the team. It is clear that the current literature on player selection in multi-player sports, especially in soccer, is very limited and scattered. The contribution of the proposed method is twofold: (1) it fills the gap in the literature with regard to quantitative applications that enable effective and efficient player selection and team formation; (2) it applies a hybrid approach that combines AHP and the 0-1 integer programming model to select soccer players.

\section{EVALUATION CRITERIA}

In general, there are six different positions in a soccer team: goalkeeper, defensive centres, fullbacks, midfielder centres, wings, and forward centres. The location of these positions on a soccer pitch is shown in Figure 1. 
It is clear that each position has different criteria. For instance, while the criterion 'tendency to punch' is very important for goalkeepers, it does not make sense if applied to forward centres. So, relevant criteria must be assigned for each position. To do so, twenty criteria are determined for each position from the famous 'Football Manager 2015' computer game. While Figure 2 summarises the criteria of each position for soccer player evaluation, each criterion in the model, and all their definitions, are provided in Table 1. As seen from Figure 2, some of the criteria are common (e.g., anticipation, agility, and first touch), while other criteria are unique to particular positions (e.g., one-on-ones and aerial ability for the goalkeeper position).

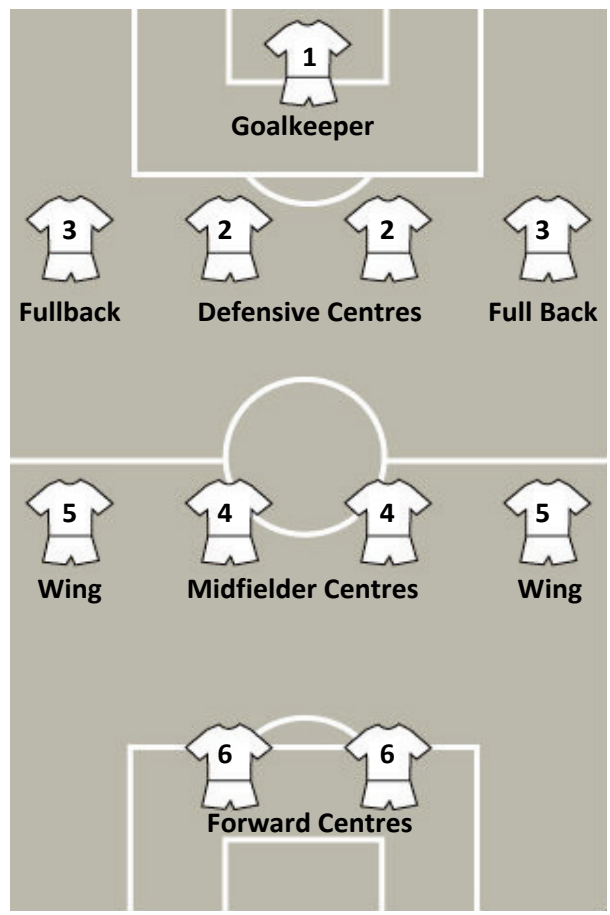

Figure 1: 4-4-2 format on a soccer pitch

Criteria for Goalkeepers
Aerial Ability
Agility
Anticipation
Bravery
Command of Area
Communication
Composure
Concentration
Decisions
Eccentricity
First Touch
Handling
Jumping
Kicking
One on Ones
Positioning
Reflexes
Rushing Out
Tendency to Punch
Throwing

Criteria for Fullbacks
Acceleration
Anticipation
Balance
Concentration
Corners
Crossing
Decisions
Dribbling
Flair
Long Throws
Marking
Natural Fitness
Off the Ball
Pace
Positioning
Stamina
Tackling
Teamwork
Technique
Work Rate

Criteria for Defensive Centres

Acceleration

Aggression

Agility

Anticipation

Balance

Bravery

Composure

Concentration

Decisions

Determination

Heading

Jumping

Leadership

Marking

Passing

Positioning

Stamina

Strength

Tackling

Teamwork 


\begin{tabular}{|l|}
\hline Criteria for Midfielder Centres \\
Acceleration \\
Aggression \\
Anticipation \\
Bravery \\
Composure \\
Decisions \\
Determination \\
Dribbling \\
First Touch \\
Flair \\
Leadership \\
Long Shots \\
Marking \\
Off the Ball \\
Passing \\
Positioning \\
Stamina \\
Strength \\
Teamwork \\
Technique \\
\hline
\end{tabular}

\begin{tabular}{|l|}
\hline Criteria for Wings \\
Acceleration \\
Agility \\
Anticipation \\
Balance \\
Crossing \\
Decisions \\
Determination \\
Dribbling \\
Finishing \\
First Touch \\
Flair \\
Long Shots \\
Natural Fitness \\
Off the Ball \\
Pace \\
Passing \\
Stamina \\
Teamwork \\
Technique \\
Work Rate \\
\hline
\end{tabular}

\begin{tabular}{|l|}
\hline Criteria for Forward Centres \\
Acceleration \\
Agility \\
Anticipation \\
Balance \\
Composure \\
Decisions \\
Determination \\
Dribbling \\
Finishing \\
First Touch \\
Flair \\
Heading \\
Jumping \\
Long Shots \\
Off the Ball \\
Pace \\
Passing \\
Stamina \\
Technique \\
Work Rate \\
\hline
\end{tabular}

Figure 2 (continues): Evaluation criteria for each position

Table 1: Definitions of each criterion [11]

\begin{tabular}{|c|c|}
\hline Criterion & Definition \\
\hline Acceleration & How fast a player can reach his/her maximum speed. \\
\hline Aerial ability & $\begin{array}{l}\text { A goalkeeper's ability to deal with the ball in aerial situations such as punching and } \\
\text { catching. }\end{array}$ \\
\hline Aggression & $\begin{array}{l}\text { A player's attitude in terms of playing mentality. An aggressive player will look to be } \\
\text { more involved in every incident and get stuck in. }\end{array}$ \\
\hline Agility & $\begin{array}{l}\text { A player's ability to start, stop, and move in different directions at varying levels of } \\
\text { speed. }\end{array}$ \\
\hline Anticipation & $\begin{array}{l}\text { A player's ability to have a sense of the unfolding events and predict what will } \\
\text { happen and react to it. }\end{array}$ \\
\hline Balance & This controls how long a player can stay on his/her feet under pressure. \\
\hline Bravery & $\begin{array}{l}\text { How committed the player is. Braver players will risk injuries in situations that less } \\
\text { brave players will shy away from. }\end{array}$ \\
\hline Command of area & $\begin{array}{l}\text { How well the goalkeeper takes charge of his/her penalty area and works with his/her } \\
\text { defensive line. }\end{array}$ \\
\hline Communication & $\begin{array}{l}\text { How well a goalkeeper communicates with his/her defensive line and organises the } \\
\text { defensive side of the team. }\end{array}$ \\
\hline Composure & A player's steadiness of mind and ability, particularly with the ball. \\
\hline Concentration & A player's concentration on an event-by-event basis. \\
\hline Corners & Accuracy of a corner kick. \\
\hline & $\begin{array}{l}\text { A player's proficiency at crossing the ball from wide into the penalty box. } \\
\text { A player's ability to make correct decisions most of the time. }\end{array}$ \\
\hline Determination & Predictions a player's commitment to success. \\
\hline Dribbling & A player's ability to dribble the ball. \\
\hline Eccentricity & $\begin{array}{l}\text { The likelihood that the goalkeeper will do the unexpected and act completely unlike } \\
\text { a goalkeeper. }\end{array}$ \\
\hline Finishing & A player's ability to put the ball in the back of the net when presented with a chance. \\
\hline First touch & $\begin{array}{l}\text { A player's ability to take control of the ball and put it into a useful position, no } \\
\text { matter how much pressure there is from the opposite team. }\end{array}$ \\
\hline Flair & $\begin{array}{l}\text { A player's ability to do the unpredicted and use his/her creativity in an attacking } \\
\text { outlet. }\end{array}$ \\
\hline Handling & $\begin{array}{l}\text { How securely the goalkeeper holds on to the ball when making a save or retrieving a } \\
\text { loose ball. }\end{array}$ \\
\hline $\begin{array}{l}\text { Heading } \\
\text { Jumping }\end{array}$ & $\begin{array}{l}\text { A player's ability to head the ball in all aerial situations. } \\
\text { How good a player is at reaching the ball in the air. }\end{array}$ \\
\hline Kicking & $\begin{array}{l}\text { The physical ability of a goalkeeper to kick the ball. This purely defines the distance } \\
\text { that the player can reach. }\end{array}$ \\
\hline Leadership & $\begin{array}{l}\text { A player's ability to influence the team and affect events or players without any } \\
\text { intentional effort. }\end{array}$ \\
\hline Long shots & $\begin{array}{l}\text { The player's prowess at shooting from a distance, such as from outside the penalty } \\
\text { area. }\end{array}$ \\
\hline $\begin{array}{l}\text { Long throws } \\
\text { Marking }\end{array}$ & $\begin{array}{l}\text { A player's ability to take long throws. } \\
\text { A player's ability to mark the opposite player. }\end{array}$ \\
\hline
\end{tabular}


Table 1 (continues): Definitions of each criterion [11]

\begin{tabular}{ll}
\hline Natural fitness & $\begin{array}{l}\text { How good the player's fitness is, and how good it stays when not training or when } \\
\text { injured. } \\
\text { Off the ball }\end{array}$ \\
$\begin{array}{l}\text { How well the player uses space when not in possession of the ball. } \\
\text { One-on-ones }\end{array}$ & $\begin{array}{l}\text { The ability of the goalkeeper to do well when faced with an opponent in a one- } \\
\text { on-one situation. }\end{array}$ \\
Pace & A player's top speed. \\
Passing & A player's ability to pass the ball. \\
Positioning & A player's ability to read situations and position himself/herself in the best possible \\
Reflexes & manner to deal with unfolding events. \\
Rushing out & How good the goalkeeper is at making instinctive reaction saves. \\
Stamina & How good the goalkeeper is at coming off his/her line to react to through balls and \\
Strength & similar situations. \\
Tackling & How well a player can endure high-level physical activity for a longer period of time. \\
Teamwork & A player's ability to exert his/her physical force on an opponent to his/her benefit. \\
Technique & How good the player will be at winning the ball cleanly without conceding fouls. \\
How well a player follows tactical instructions and works for the team. & \\
Tendency to punch & A player's aesthetic quality when passing the ball, shooting, or crossing; or how \\
Throwing & Whether a player will be with the ball. \\
Work rate & How good the goalkeeper's distribution of the ball with his/her arms is. \\
\hline
\end{tabular}

\section{THE ANALYTIC HIERARCHIC PROCESS FOR ESTIMATING ATTRIBUTE WEIGHTS}

The Analytic Hierarchy Process (AHP) method was developed by Saaty [12]. AHP assumes that evaluation criteria can be completely expressed in a hierarchical structure. The data acquired from the decision-makers is pairwise comparisons of the relative importance of each of the criteria, or the degree of preference of one factor over another with respect to each criterion [13]. AHP has the advantage of permitting a hierarchical structure of the criteria, which provides users with a better focus on specific criteria and sub-criteria when allocating the weights. The AHP modelling process involves three phases: structuring the decision problem, pairwise comparison, and calculating the priorities of each criterion. Using this three-phase approach, the priorities of the criteria for each position that will be an input for the mathematical model are obtained. It is noted that AHP is used to find only the priorities of each criterion, not to select the best soccer player among alternatives. Selection will be done by a mathematical model that will be given in the next section.

\subsection{Structuring the decision problem}

There are six different goals in the decision problem: to select the best soccer players for the six different positions - goalkeepers, defensive centres, fullbacks, midfielder centres, wings, and forward centres. This phase involves formulating an appropriate hierarchy of the AHP model that consists of the goal and the criteria. As seen in Figure 2 above, there are twenty criteria for each goal.

\subsection{Pairwise comparison}

After building the AHP hierarchy, the next phase is the pairwise comparison, which involves forming a team of experts. The nine-point scale, as shown in Table 2 , is used to assign pairwise comparisons of all criteria in each position of the hierarchy.

Table 2: The fundamental scale of absolute numbers [14]

\begin{tabular}{|c|c|c|}
\hline $\begin{array}{l}\text { Intensity of } \\
\text { Importance }\end{array}$ & Definition & Explanation \\
\hline 1 & Equal importance & \multirow{2}{*}{$\begin{array}{l}\text { Two criteria contribute equally to the objective } \\
\text { Experience and judgment slightly favour one } \\
\text { criterion over another }\end{array}$} \\
\hline 3 & Moderate importance & \\
\hline 5 & Strong importance & $\begin{array}{l}\text { Experience and judgment strongly favour one } \\
\text { criterion over another }\end{array}$ \\
\hline 7 & Very strong importance & $\begin{array}{l}\text { A criterion is favoured very strongly over another; its } \\
\text { dominance is demonstrated in practice }\end{array}$ \\
\hline 9 & Extreme importance & $\begin{array}{l}\text { The evidence favouring one criterion over another is } \\
\text { of the highest possible order of affirmation }\end{array}$ \\
\hline
\end{tabular}


Table 2 (continues): The fundamental scale of absolute numbers [14]

\begin{tabular}{|c|c|c|}
\hline $2,4,6,8$ & $\begin{array}{l}\text { For compromise between the } \\
\text { above values }\end{array}$ & $\begin{array}{l}\text { Sometimes one needs to interpolate a compromise } \\
\text { judgment numerically because there is no good word } \\
\text { to describe it }\end{array}$ \\
\hline $\begin{array}{l}\text { Reciprocals } \\
\text { of above }\end{array}$ & $\begin{array}{l}\text { If criterion } i \text { has one of the } \\
\text { above non-zero numbers } \\
\text { assigned to it when compared } \\
\text { with criterion } j \text {, then } j \text { has } \\
\text { the reciprocal value when } \\
\text { compared with } i\end{array}$ & A reasonable assumption \\
\hline
\end{tabular}

Usually, every member assigns his/her pairwise comparisons, which will be translated into the corresponding pairwise comparison judgment matrices. As suggested by Saaty [14], the geometric mean approach is used to combine the individual judgment matrices to obtain the consensus for the entire expert group. The expert group consists of three academicians from the Physical Education and Sports School at the Gaziantep University; these academics have lots of information on and expertise in soccer and soccer players. Face-to-face interviews were used to collect the pairwise comparison judgments from the experts. Tables 3 to 8 give pairwise comparison matrices of the main criteria for particular positions. It is noted that the geometric means of the three experts were rounded up.

\subsection{Calculating the priorities}

After the pairwise comparison, the last step is calculating the priorities of each criterion. The software system called Expert Choice 11.0 was used to determine the priority weights; the obtained priority weights are given in Tables 9 and 10. The consistency ratio (CR) of each position is also shown below each table. It should be noted that the quality of the output of the AHP is strictly related to the consistency of the pairwise comparison judgments. It can be seen that the consistency ratio of each of the pairwise comparisons is equal to or less than 0.04 , which is well below the ruleof-thumb value of $C R=0.1$. This clearly implies that the evaluators are consistent in assigning pairwise comparison judgments. It is noted that the AHP is based on the fundamental assumption of preferential independence between the decision criteria shown in Figure 2. Details about the various steps of AHP, and especially the mathematical background of AHP, can be found in the literature $[15,16]$; free and open source software can also be downloaded from http://expertchoice.com/.

\section{MATHEMATICAL MODEL FOR SOCCER PLAYER SELECTION}

The proposed mathematical model for soccer player selection is introduced in this section.

\section{Sets and Indices}

$\mathrm{P} \quad$ is the set of all players. The index $\mathrm{j}$ will be used as the index for the set of players

$\mathrm{G} \quad$ is the set of goalkeepers. Note that $\mathrm{G} \in \mathrm{P}$

$\mathrm{D} \quad$ is the set of defensive centre players. Note that $\mathrm{D} \in \mathrm{P}$

$\mathrm{B}$ is the set of fullback players. Note that $\mathrm{B} \in \mathrm{P}$

$\mathrm{M}$ is the set of midfielder centre players. Note that $\mathrm{M} \in \mathrm{P}$

$\mathrm{W}$ is the set of wing players. Note that $\mathrm{W} \in \mathrm{P}$

$\mathrm{F} \quad$ is the set of forward centre players. Note that $\mathrm{F} \in \mathrm{P}$

$\mathrm{S} \quad$ is the set of foreign players. Note that $\mathrm{S} \in \mathrm{P}$

$\mathrm{R}$ is the set of positions in the team. 1 is defined as a goalkeeper, 2 as a defensive centre, 3 as a fullback, 4 as a midfielder centre, 5 as a wing, and 6 as a forward centre. The index $i$ will be used as the index for the set of positions.

$\mathrm{C}$ is the set of criteria. The index c will be used as the index for the set of criteria.

\section{Variables}

$\mathrm{X}_{\mathrm{ji}} \quad$ A binary variable equal to 1 if player $\mathrm{j} \in \mathrm{P}$ is assigned to position $\mathrm{i} \in \mathrm{R}$; otherwise it is 0 
Table 3: Pairwise comparison matrix for the criteria specific to the goalkeeper

\begin{tabular}{|c|c|c|c|c|c|c|c|c|c|c|c|c|c|c|c|c|c|c|c|c|c|c|}
\hline & C1 & C2 & $\mathrm{C} 3$ & C4 & C5 & C6 & C7 & C8 & C9 & C10 & C11 & C12 & C13 & C14 & C15 & C16 & C17 & C18 & C19 & $\mathrm{C20}$ & & Criteria \\
\hline C1 & 1 & 3 & 1 & $1 / 2$ & 5 & 1 & 2 & 1 & 4 & $1 / 3$ & $1 / 4$ & 1 & 4 & $1 / 5$ & $1 / 4$ & $1 / 3$ & $1 / 3$ & 2 & 1 & $1 / 2$ & C1 & Rushing Out \\
\hline C2 & & 1 & $1 / 2$ & $1 / 5$ & $1 / 5$ & $1 / 3$ & 1 & $1 / 5$ & 2 & $1 / 7$ & $1 / 8$ & $1 / 2$ & 2 & $1 / 5$ & $1 / 5$ & $1 / 6$ & $1 / 8$ & $1 / 2$ & $1 / 3$ & $1 / 6$ & $\mathrm{C} 2$ & Kicking \\
\hline C3 & & & 1 & 1 & 3 & 1 & 1 & 2 & 3 & $1 / 5$ & $1 / 3$ & 1 & 4 & $1 / 4$ & $1 / 3$ & $1 / 4$ & $1 / 5$ & 1 & $1 / 3$ & $1 / 4$ & C3 & One on Ones \\
\hline C4 & & & & 1 & 3 & 1 & 1 & 4 & 5 & $1 / 2$ & $1 / 5$ & 1 & 5 & $1 / 5$ & $1 / 4$ & $1 / 4$ & $1 / 3$ & 1 & $1 / 4$ & $1 / 5$ & C4 & Command of Area \\
\hline C5 & & & & & 1 & $1 / 3$ & $1 / 5$ & 2 & 3 & $1 / 6$ & $1 / 7$ & $1 / 3$ & 4 & $1 / 5$ & $1 / 6$ & $1 / 7$ & $1 / 8$ & $1 / 3$ & $1 / 4$ & $1 / 5$ & C5 & Eccentricity \\
\hline C6 & & & & & & 1 & $1 / 2$ & 4 & 6 & $1 / 3$ & $1 / 3$ & 1 & 6 & $1 / 2$ & $1 / 3$ & $1 / 4$ & $1 / 5$ & 1 & $1 / 2$ & $1 / 2$ & C6 & Handling \\
\hline C7 & & & & & & & 1 & 5 & 7 & $1 / 3$ & $1 / 3$ & 3 & 6 & 1 & $1 / 3$ & $1 / 5$ & $1 / 4$ & 3 & 1 & 1 & C7 & Aerial Ability \\
\hline C8 & & & & & & & & 1 & 3 & $1 / 7$ & $1 / 7$ & $1 / 3$ & 2 & $1 / 5$ & $1 / 9$ & $1 / 8$ & $1 / 7$ & $1 / 3$ & $1 / 5$ & $1 / 5$ & $\mathrm{C} 8$ & Communication \\
\hline C9 & & & & & & & & & 1 & $1 / 6$ & $1 / 6$ & $1 / 5$ & $1 / 2$ & $1 / 6$ & $1 / 8$ & $1 / 8$ & $1 / 7$ & $1 / 5$ & $1 / 5$ & $1 / 4$ & C9 & First Touch \\
\hline C10 & & & & & & & & & & 1 & 1 & 2 & 4 & 1 & $1 / 2$ & $1 / 2$ & 1 & 2 & 2 & 2 & C10 & Reflexes \\
\hline C11 & & & & & & & & & & & 1 & 3 & 5 & 1 & $1 / 2$ & $1 / 2$ & 1 & 3 & 3 & 3 & C11 & Throwing \\
\hline C12 & & & & & & & & & & & & 1 & 3 & $1 / 5$ & $1 / 7$ & $1 / 7$ & $1 / 3$ & 1 & 1 & 1 & $\mathrm{C} 12$ & Tendency to Punch \\
\hline C13 & & & & & & & & & & & & & 1 & $1 / 6$ & $1 / 7$ & $1 / 7$ & $1 / 8$ & $1 / 3$ & $1 / 3$ & $1 / 2$ & C13 & Bravery \\
\hline C14 & & & & & & & & & & & & & & 1 & 2 & $1 / 2$ & $1 / 2$ & 3 & 2 & 3 & C14 & Decisions \\
\hline C15 & & & & & & & & & & & & & & & 1 & 1 & 1 & 6 & 4 & 4 & C15 & Concentration \\
\hline C16 & & & & & & & & & & & & & & & & 1 & 1 & 4 & 3 & 2 & C16 & Anticipation \\
\hline C17 & & & & & & & & & & & & & & & & & 1 & 4 & 3 & 2 & C17 & Positioning \\
\hline C18 & & & & & & & & & & & & & & & & & & 1 & $1 / 3$ & $1 / 2$ & C18 & Composure \\
\hline C19 & & & & & & & & & & & & & & & & & & & 1 & 1 & C19 & Agility \\
\hline C20 & & & & & & & & & & & & & & & & & & & & 1 & $\mathrm{C} 20$ & Jumping \\
\hline
\end{tabular}

Table 4: Pairwise comparison matrix for the criteria specific to defensive centres

\begin{tabular}{|c|c|c|c|c|c|c|c|c|c|c|c|c|c|c|c|c|c|c|c|c|c|c|}
\hline & $\mathrm{C} 1$ & $\mathrm{C2}$ & $\mathrm{C3}$ & $\mathrm{C4}$ & C5 & $\mathrm{C} 6$ & C7 & $\mathrm{C} 8$ & C9 & $\mathrm{C} 10$ & C11 & C12 & C13 & C14 & C15 & C16 & C17 & C18 & C19 & $\mathrm{C20}$ & & Criteria \\
\hline C1 & 1 & $1 / 3$ & 4 & $1 / 6$ & $1 / 3$ & 3 & 6 & 6 & $1 / 3$ & 2 & $1 / 5$ & $1 / 5$ & $1 / 3$ & 4 & 1 & $1 / 3$ & 3 & 1 & 2 & $1 / 2$ & C1 & Heading \\
\hline C2 & & 1 & 5 & $1 / 2$ & 1 & 2 & 3 & 3 & 1 & 2 & $1 / 2$ & $1 / 2$ & 1 & 3 & 3 & 1 & 4 & 2 & 6 & 3 & C2 & Marking \\
\hline C3 & & & 1 & $1 / 6$ & $1 / 3$ & $1 / 3$ & $1 / 2$ & $1 / 2$ & $1 / 5$ & $1 / 4$ & $1 / 8$ & $1 / 5$ & $1 / 5$ & $1 / 3$ & 1 & $1 / 4$ & 2 & $1 / 3$ & 3 & $1 / 4$ & C3 & Passing \\
\hline C4 & & & & 1 & 3 & 3 & 4 & 4 & 1 & 1 & $1 / 3$ & 1 & 1 & 1 & 4 & 1 & 6 & 2 & 7 & 3 & C4 & Tackling \\
\hline C5 & & & & & 1 & 1 & 2 & 2 & $1 / 4$ & $1 / 5$ & $1 / 8$ & $1 / 3$ & $1 / 3$ & $1 / 4$ & 1 & $1 / 5$ & 2 & 1 & 3 & 1 & C5 & Aggression \\
\hline C6 & & & & & & 1 & 2 & 2 & $1 / 5$ & $1 / 4$ & $1 / 7$ & $1 / 5$ & $1 / 5$ & $1 / 6$ & 1 & $1 / 5$ & 3 & 1 & 3 & 1 & C6 & Bravery \\
\hline C7 & & & & & & & 1 & 1 & $1 / 6$ & $1 / 7$ & $1 / 8$ & $1 / 6$ & $1 / 7$ & $1 / 5$ & $1 / 2$ & $1 / 5$ & 3 & 1 & 3 & $1 / 2$ & C7 & Decisions \\
\hline C8 & & & & & & & & 1 & $1 / 5$ & $1 / 5$ & $1 / 6$ & $1 / 6$ & $1 / 5$ & $1 / 3$ & $1 / 5$ & $1 / 4$ & 3 & 1 & 3 & $1 / 2$ & $\mathrm{C} 8$ & Determination \\
\hline C9 & & & & & & & & & 1 & 1 & 1 & $1 / 2$ & 1 & $1 / 2$ & 1 & 2 & 6 & 4 & 5 & 3 & C9 & Concentration \\
\hline C10 & & & & & & & & & & 1 & 1 & $1 / 2$ & 1 & 1 & 1 & 3 & 5 & 4 & 5 & 3 & C10 & Leadership \\
\hline C11 & & & & & & & & & & & 1 & $1 / 2$ & 1 & 1 & $1 / 2$ & 3 & 5 & 4 & 4 & 1 & C11 & Anticipation \\
\hline C12 & & & & & & & & & & & & 1 & 2 & 4 & 1 & 5 & 7 & 6 & 5 & 4 & C12 & Positioning \\
\hline C13 & & & & & & & & & & & & & 1 & 3 & $1 / 3$ & 3 & 5 & 4 & 3 & 3 & C13 & Composure \\
\hline C14 & & & & & & & & & & & & & & 1 & $1 / 6$ & $1 / 3$ & 2 & 2 & 1 & $1 / 6$ & C14 & Teamwork \\
\hline C15 & & & & & & & & & & & & & & & 1 & 5 & 6 & 5 & 3 & 4 & C15 & Agility \\
\hline C16 & & & & & & & & & & & & & & & & 1 & 2 & 1 & $1 / 3$ & $1 / 4$ & C16 & Stamina \\
\hline C17 & & & & & & & & & & & & & & & & & 1 & $1 / 4$ & $1 / 6$ & $1 / 7$ & C17 & Balance \\
\hline C18 & & & & & & & & & & & & & & & & & & 1 & 2 & 1 & C18 & Strength \\
\hline C19 & & & & & & & & & & & & & & & & & & & 1 & $1 / 3$ & C19 & Acceleration \\
\hline $\mathrm{C} 20$ & & & & & & & & & & & & & & & & & & & & 1 & $\mathrm{C} 20$ & Jumping \\
\hline
\end{tabular}


Table 5: Pairwise comparison matrix for the criteria specific to fullbacks

\begin{tabular}{|c|c|c|c|c|c|c|c|c|c|c|c|c|c|c|c|c|c|c|c|c|c|c|}
\hline & C1 & C2 & C3 & C4 & C5 & C6 & C7 & C8 & C9 & C10 & C11 & C12 & C13 & C14 & C15 & C16 & C17 & C18 & C19 & $\mathrm{C20}$ & & Criteria \\
\hline C1 & 1 & 5 & $1 / 3$ & 1 & 2 & $1 / 2$ & 3 & 1 & 2 & 4 & 3 & 1 & 2 & 1 & 2 & 3 & 3 & $1 / 3$ & $1 / 5$ & $1 / 2$ & C1 & Dribbling \\
\hline C2 & & 1 & $1 / 5$ & $1 / 4$ & $1 / 2$ & $1 / 6$ & $1 / 2$ & $1 / 5$ & $1 / 3$ & 1 & $1 / 2$ & $1 / 5$ & $1 / 8$ & $1 / 3$ & $1 / 2$ & $1 / 6$ & $1 / 2$ & $1 / 7$ & $1 / 8$ & $1 / 5$ & C2 & Corners \\
\hline C3 & & & 1 & 2 & 3 & 1 & 4 & 1 & 2 & 5 & 3 & 1 & $1 / 2$ & 2 & 5 & $1 / 3$ & 3 & $1 / 3$ & $1 / 3$ & 1 & C3 & Marking \\
\hline C4 & & & & & 3 & 1 & 3 & 2 & 2 & 4 & 2 & 2 & 2 & 3 & 3 & 4 & 2 & $1 / 2$ & 1 & $1 / 3$ & C4 & Crossing \\
\hline C5 & & & & & 1 & 3 & 1 & 4 & 2 & 2 & 1 & 3 & 4 & 2 & 1 & 3 & 2 & 4 & 6 & 4 & C5 & Technique \\
\hline C6 & & & & & & 1 & 5 & 2 & 1 & 4 & 3 & 1 & 2 & 3 & 6 & 1 & 3 & 1 & 1 & 1 & $\mathrm{C} 6$ & Tackling \\
\hline C7 & & & & & & & 1 & $1 / 3$ & $1 / 6$ & 1 & $1 / 2$ & $1 / 6$ & $1 / 2$ & $1 / 2$ & 2 & $1 / 5$ & $1 / 2$ & $1 / 4$ & $1 / 3$ & $1 / 4$ & C7 & Long Throws \\
\hline C8 & & & & & & & & 1 & $1 / 3$ & 4 & 1 & $1 / 3$ & 2 & 2 & 6 & 1 & 2 & 1 & 1 & $1 / 2$ & $\mathrm{C} 8$ & Work Rate \\
\hline C9 & & & & & & & & & 1 & 6 & 4 & 1 & 3 & 3 & 7 & 3 & 4 & 3 & 3 & 2 & C9 & Decisions \\
\hline C10 & & & & & & & & & & 1 & $1 / 3$ & $1 / 6$ & $1 / 4$ & $1 / 4$ & 2 & $1 / 3$ & $1 / 4$ & $1 / 3$ & $1 / 3$ & $1 / 6$ & C10 & Concentration \\
\hline C11 & & & & & & & & & & & 1 & $1 / 3$ & 1 & 1 & 4 & 1 & 1 & 1 & 1 & $1 / 2$ & C11 & Anticipation \\
\hline C12 & & & & & & & & & & & & 1 & 3 & 3 & 6 & 4 & 4 & 3 & 4 & 2 & C12 & Flair \\
\hline C13 & & & & & & & & & & & & & 1 & 1 & 3 & 2 & 1 & 1 & 2 & $1 / 2$ & C13 & Positioning \\
\hline C14 & & & & & & & & & & & & & & 1 & 3 & 2 & 1 & 1 & 3 & $1 / 3$ & C14 & Teamwork \\
\hline C15 & & & & & & & & & & & & & & & 1 & $1 / 3$ & $1 / 3$ & $1 / 3$ & 1 & $1 / 9$ & C15 & Off the Ball \\
\hline C16 & & & & & & & & & & & & & & & & 1 & 1 & 1 & 4 & $1 / 3$ & C16 & Stamina \\
\hline C17 & & & & & & & & & & & & & & & & & 1 & 1 & $1 / 4$ & 1 & C17 & Balance \\
\hline C18 & & & & & & & & & & & & & & & & & & 1 & 3 & 4 & C18 & Pace \\
\hline C19 & & & & & & & & & & & & & & & & & & & 1 & 2 & C19 & Acceleration \\
\hline C20 & & & & & & & & & & & & & & & & & & & & 1 & $\mathrm{C} 20$ & Natural Fitness \\
\hline
\end{tabular}

Table 6: Pairwise comparison matrix for the criteria specific to midfielder centres

\begin{tabular}{|c|c|c|c|c|c|c|c|c|c|c|c|c|c|c|c|c|c|c|c|c|c|c|}
\hline & $\mathrm{C} 1$ & $\mathrm{C} 2$ & $\mathrm{C3}$ & $\mathrm{C4}$ & C5 & $\mathrm{C} 6$ & $\mathrm{C7}$ & $\mathrm{C} 8$ & C9 & $\mathrm{C} 10$ & C11 & $\mathrm{C} 12$ & C13 & $\mathrm{C} 14$ & C15 & C16 & C17 & C18 & C19 & $\mathrm{C} 20$ & & Criteria \\
\hline C1 & 1 & 4 & 2 & $1 / 4$ & $1 / 3$ & 1 & 3 & 2 & $1 / 4$ & 3 & $1 / 2$ & $1 / 3$ & $1 / 2$ & $1 / 2$ & 1 & $1 / 3$ & $1 / 5$ & 2 & 1 & 2 & C1 & Dribbling \\
\hline C2 & & 1 & $1 / 2$ & $1 / 4$ & $1 / 7$ & $1 / 5$ & $1 / 2$ & $1 / 3$ & $1 / 4$ & $1 / 4$ & $1 / 6$ & $1 / 7$ & $1 / 6$ & $1 / 6$ & $1 / 3$ & $1 / 7$ & $1 / 5$ & $1 / 2$ & $1 / 2$ & $1 / 2$ & $\mathrm{C} 2$ & First Touch \\
\hline C3 & & & 1 & $1 / 2$ & $1 / 3$ & $1 / 4$ & 1 & $1 / 2$ & $1 / 2$ & $1 / 3$ & $1 / 2$ & $1 / 3$ & $1 / 4$ & $1 / 4$ & $1 / 2$ & $1 / 6$ & $1 / 3$ & 1 & 1 & $1 / 2$ & $\mathrm{C} 3$ & Marking \\
\hline C4 & & & & 1 & $1 / 2$ & $1 / 3$ & 3 & 1 & 2 & $1 / 2$ & 1 & $1 / 2$ & $1 / 4$ & $1 / 3$ & 1 & $1 / 5$ & $1 / 3$ & 3 & 2 & 1 & C4 & Passing \\
\hline C5 & & & & & 1 & $1 / 2$ & 6 & 2 & 4 & 1 & 2 & 1 & $1 / 3$ & $1 / 2$ & 2 & $1 / 5$ & $1 / 2$ & 5 & 4 & 2 & C5 & Technique \\
\hline C6 & & & & & & 1 & 7 & 4 & 6 & 2 & 4 & 2 & $1 / 2$ & 1 & 4 & $1 / 4$ & 1 & $1 / 8$ & $1 / 6$ & $1 / 4$ & $\mathrm{C} 6$ & Long Shots \\
\hline C7 & & & & & & & 1 & $1 / 3$ & $1 / 4$ & $1 / 4$ & $1 / 2$ & $1 / 5$ & $1 / 8$ & $1 / 5$ & $1 / 6$ & $1 / 7$ & $1 / 5$ & $1 / 8$ & $1 / 8$ & $1 / 5$ & C7 & Aggression \\
\hline C8 & & & & & & & & 1 & 1 & 1 & 2 & $1 / 2$ & $1 / 4$ & $1 / 2$ & $1 / 2$ & $1 / 3$ & 1 & $1 / 3$ & $1 / 3$ & 1 & $\mathrm{C} 8$ & Bravery \\
\hline C9 & & & & & & & & & 1 & 1 & 1 & $1 / 3$ & $1 / 4$ & $1 / 2$ & 3 & $1 / 4$ & 1 & $1 / 5$ & $1 / 4$ & $1 / 4$ & C9 & Decisions \\
\hline C10 & & & & & & & & & & 1 & 4 & $1 / 3$ & $1 / 4$ & $1 / 2$ & $1 / 2$ & $1 / 4$ & 1 & $1 / 2$ & $1 / 5$ & $1 / 2$ & C10 & Determination \\
\hline C11 & & & & & & & & & & & 1 & $1 / 5$ & $1 / 7$ & $1 / 4$ & $1 / 4$ & $1 / 6$ & $1 / 4$ & $1 / 5$ & $1 / 7$ & $1 / 3$ & C11 & Leadership \\
\hline C12 & & & & & & & & & & & & 1 & $1 / 2$ & 2 & $1 / 2$ & $1 / 3$ & 2 & 1 & $1 / 3$ & 2 & C12 & Anticipation \\
\hline C13 & & & & & & & & & & & & & 1 & 4 & $1 / 4$ & 1 & 4 & 2 & 1 & 5 & C13 & Flair \\
\hline C14 & & & & & & & & & & & & & & 1 & 1 & $1 / 4$ & 1 & $1 / 2$ & $1 / 5$ & 2 & C14 & Positioning \\
\hline C15 & & & & & & & & & & & & & & & 1 & $1 / 6$ & 1 & $1 / 3$ & $1 / 5$ & 3 & C15 & Composure \\
\hline C16 & & & & & & & & & & & & & & & & 1 & 5 & 2 & 1 & 5 & C16 & Teamwork \\
\hline C17 & & & & & & & & & & & & & & & & & 1 & $1 / 4$ & $1 / 5$ & 1 & C17 & Off the Ball \\
\hline C18 & & & & & & & & & & & & & & & & & & 1 & 1 & 3 & C18 & Stamina \\
\hline C19 & & & & & & & & & & & & & & & & & & & 1 & 1 & C19 & Strength \\
\hline C20 & & & & & & & & & & & & & & & & & & & & 1 & $\mathrm{C} 20$ & Acceleration \\
\hline
\end{tabular}


Table 7: Pairwise comparison matrix for the criteria specific to wings

\begin{tabular}{|c|c|c|c|c|c|c|c|c|c|c|c|c|c|c|c|c|c|c|c|c|c|c|}
\hline & C1 & $\mathrm{C} 2$ & $\mathrm{C3}$ & C4 & C5 & C6 & C7 & C8 & C9 & C10 & C11 & C12 & C13 & C14 & C15 & C16 & C17 & C18 & C19 & $\mathrm{C} 20$ & & Criteria \\
\hline C1 & 1 & $1 / 5$ & 1 & $1 / 6$ & $1 / 3$ & $1 / 5$ & $1 / 2$ & $1 / 3$ & 2 & 2 & $1 / 2$ & $1 / 4$ & $1 / 2$ & $1 / 5$ & $1 / 4$ & $1 / 2$ & $1 / 3$ & $1 / 5$ & $1 / 5$ & $1 / 4$ & C1 & Finishing \\
\hline C2 & & 1 & 5 & 1 & 2 & 1 & 3 & 2 & 6 & 5 & 1 & 1 & 2 & 1 & 2 & 1 & 2 & 1 & 1 & 1 & C2 & Dribbling \\
\hline C3 & & & 1 & $1 / 3$ & $1 / 3$ & $1 / 6$ & $1 / 2$ & $1 / 2$ & 2 & 2 & $1 / 4$ & $1 / 4$ & $1 / 3$ & $1 / 4$ & $1 / 3$ & 4 & $1 / 3$ & $1 / 4$ & $1 / 4$ & $1 / 3$ & C3 & First Touch \\
\hline C4 & & & & 1 & 1 & $1 / 3$ & 2 & 2 & 4 & 4 & $1 / 2$ & $1 / 2$ & 1 & $1 / 2$ & 1 & 2 & 1 & $1 / 2$ & $1 / 2$ & 1 & C4 & Crossing \\
\hline C5 & & & & & 1 & $1 / 2$ & 2 & 2 & 4 & 4 & $1 / 2$ & $1 / 2$ & 1 & $1 / 2$ & 1 & $1 / 2$ & 1 & $1 / 2$ & $1 / 2$ & 1 & C5 & Passing \\
\hline C6 & & & & & & 1 & 2 & 2 & 3 & 5 & $1 / 2$ & $1 / 2$ & 1 & $1 / 2$ & 1 & $1 / 2$ & 1 & $1 / 2$ & $1 / 2$ & 1 & C6 & Technique \\
\hline C7 & & & & & & & 1 & 1 & 2 & 3 & $1 / 4$ & $1 / 4$ & $1 / 2$ & $1 / 5$ & $1 / 3$ & $1 / 5$ & 1 & $1 / 6$ & $1 / 5$ & $1 / 3$ & C7 & Long Shots \\
\hline C8 & & & & & & & & 1 & 3 & 3 & $1 / 4$ & $1 / 4$ & $1 / 2$ & $1 / 6$ & $1 / 4$ & $1 / 5$ & 1 & $1 / 6$ & $1 / 5$ & $1 / 5$ & $\mathrm{C} 8$ & Work Rate \\
\hline C9 & & & & & & & & & 1 & 1 & $1 / 6$ & $1 / 6$ & $1 / 3$ & $1 / 8$ & $1 / 5$ & $1 / 4$ & $1 / 2$ & $1 / 4$ & $1 / 4$ & $1 / 3$ & C9 & Decisions \\
\hline C10 & & & & & & & & & & 1 & $1 / 4$ & $1 / 5$ & $1 / 3$ & $1 / 6$ & $1 / 6$ & $1 / 5$ & $1 / 3$ & $1 / 5$ & $1 / 5$ & $1 / 4$ & C10 & Determination \\
\hline C11 & & & & & & & & & & & 1 & 1 & 2 & $1 / 2$ & $1 / 2$ & 1 & 3 & 1 & 1 & 2 & C11 & Anticipation \\
\hline C12 & & & & & & & & & & & & 1 & 3 & $1 / 2$ & $1 / 3$ & 1 & 3 & 1 & 2 & 3 & C12 & Flair \\
\hline C13 & & & & & & & & & & & & & 1 & $1 / 5$ & $1 / 6$ & $1 / 3$ & 1 & $1 / 3$ & $1 / 2$ & 1 & C13 & Teamwork \\
\hline C14 & & & & & & & & & & & & & & 1 & 1 & 2 & 5 & 2 & 3 & 4 & C14 & Off the Ball \\
\hline C15 & & & & & & & & & & & & & & & 1 & 2 & 5 & 2 & 3 & 4 & C15 & Agility \\
\hline C16 & & & & & & & & & & & & & & & & 1 & 3 & 1 & 2 & 3 & C16 & Stamina \\
\hline C17 & & & & & & & & & & & & & & & & & 1 & $1 / 4$ & $1 / 2$ & 1 & C17 & Balance \\
\hline C18 & & & & & & & & & & & & & & & & & & 1 & 3 & 4 & C18 & Pace \\
\hline C19 & & & & & & & & & & & & & & & & & & & 1 & 1 & C19 & Acceleration \\
\hline C20 & & & & & & & & & & & & & & & & & & & & 1 & $\mathrm{C} 20$ & Natural Fitness \\
\hline
\end{tabular}

Table 8: Pairwise comparison matrix for the criteria specific to forward centres

\begin{tabular}{|c|c|c|c|c|c|c|c|c|c|c|c|c|c|c|c|c|c|c|c|c|c|c|}
\hline & C1 & C2 & C3 & C4 & C5 & C6 & C7 & C8 & C9 & C10 & C11 & C12 & C13 & C14 & C15 & C16 & C17 & C18 & C19 & $\mathrm{C20}$ & & Criteria \\
\hline C1 & 1 & 6 & 3 & 2 & 4 & 2 & 3 & 2 & 2 & 3 & 3 & 2 & 2 & 2 & 4 & 3 & 4 & 2 & 3 & 4 & $\mathrm{C1}$ & Finishing \\
\hline C2 & & 1 & $1 / 5$ & $1 / 4$ & $1 / 2$ & $1 / 5$ & $1 / 2$ & $1 / 3$ & $1 / 7$ & $1 / 2$ & $1 / 6$ & $1 / 6$ & $1 / 3$ & $1 / 6$ & $1 / 8$ & $1 / 2$ & $1 / 4$ & $1 / 3$ & $1 / 7$ & $1 / 4$ & $\mathrm{C} 2$ & Dribbling \\
\hline C3 & & & 1 & 2 & 3 & 1 & 3 & 1 & $1 / 3$ & 2 & $1 / 2$ & 1 & 2 & $1 / 2$ & 2 & 3 & 1 & 2 & 2 & 2 & $\mathrm{C} 3$ & First Touch \\
\hline C4 & & & & 1 & 5 & $1 / 3$ & 4 & 1 & 44 & 5 & $1 / 3$ & $1 / 2$ & 3 & $1 / 3$ & 1 & 2 & $1 / 2$ & 1 & 1 & 3 & C4 & Heading \\
\hline C5 & & & & & 1 & $1 / 4$ & 1 & $1 / 3$ & $1 / 3$ & $1 / 2$ & $1 / 5$ & $1 / 4$ & $1 / 2$ & $1 / 6$ & $1 / 6$ & 1 & $1 / 4$ & $1 / 2$ & $1 / 5$ & $1 / 4$ & C5 & Passing \\
\hline C6 & & & & & & 1 & 5 & 2 & 3 & 3 & 1 & 1 & 2 & $1 / 2$ & 4 & 4 & 1 & 2 & 2 & 4 & C6 & Technique \\
\hline C7 & & & & & & & 1 & $1 / 3$ & $1 / 6$ & $1 / 2$ & $1 / 4$ & $1 / 4$ & $1 / 2$ & $1 / 5$ & $1 / 8$ & 1 & $1 / 4$ & $1 / 2$ & $1 / 6$ & $1 / 5$ & C7 & Long Shots \\
\hline C8 & & & & & & & & 1 & $1 / 3$ & 2 & $1 / 2$ & $1 / 2$ & 2 & $1 / 2$ & 1 & 3 & $1 / 2$ & 2 & 2 & $1 / 2$ & C8 & Work Rate \\
\hline C9 & & & & & & & & & 1 & 3 & $1 / 4$ & $1 / 5$ & 3 & $1 / 3$ & 1 & 2 & 3 & 1 & 1 & 3 & C9 & Decisions \\
\hline C10 & & & & & & & & & & 1 & $1 / 4$ & $1 / 5$ & 1 & $1 / 4$ & $1 / 5$ & 3 & $1 / 4$ & 1 & $1 / 5$ & $1 / 3$ & C10 & Determination \\
\hline C11 & & & & & & & & & & & 1 & 1 & 4 & 1 & 4 & 7 & 1 & 4 & 4 & 3 & C11 & Anticipation \\
\hline C12 & & & & & & & & & & & & 1 & 4 & 1 & 5 & 7 & 1 & 4 & 4 & 4 & $\mathrm{C} 12$ & Flair \\
\hline C13 & & & & & & & & & & & & & 1 & $1 / 4$ & $1 / 8$ & 2 & $1 / 4$ & 1 & 1 & 2 & C13 & Composure \\
\hline C14 & & & & & & & & & & & & & & 1 & 5 & 5 & 1 & 4 & 4 & 2 & C14 & Off the Ball \\
\hline C15 & & & & & & & & & & & & & & & 1 & 1 & $1 / 4$ & 1 & $1 / 3$ & 1 & C15 & Agility \\
\hline C16 & & & & & & & & & & & & & & & & 1 & $1 / 5$ & 1 & $1 / 3$ & 1 & C16 & Stamina \\
\hline C17 & & & & & & & & & & & & & & & & & 1 & 5 & 3 & 1 & C17 & Balance \\
\hline C18 & & & & & & & & & & & & & & & & & & 1 & $1 / 3$ & 2 & C18 & Pace \\
\hline C19 & & & & & & & & & & & & & & & & & & & 1 & 2 & C19 & Acceleration \\
\hline C20 & & & & & & & & & & & & & & & & & & & & 1 & $\mathrm{C} 20$ & Jumping \\
\hline
\end{tabular}


Table 9: Priority weights for goalkeepers, defensive centres, and fullbacks

\begin{tabular}{llllll}
\hline Goalkeeper & & Defensive Centres & & Fullbacks & Priority \\
\hline Criteria & Priority & Criteria & Priority & Criteria & 0.057 \\
Rushing Out & 0.034 & Heading & 0.043 & Dribbling & 0.012 \\
Kicking & 0.014 & Marking & 0.069 & Corners & 0.070 \\
One on Ones & 0.027 & Passing & 0.016 & Marking & 0.072 \\
Command of Area & 0.034 & Tackling & 0.084 & Crossing & 0.097 \\
Eccentricity & 0.017 & Aggression & 0.031 & Technique & 0.063 \\
Handling & 0.033 & Bravery & 0.024 & Tackling & 0.018 \\
Aerial Ability & 0.045 & Decisions & 0.017 & Long Throws & 0.042 \\
Communication & 0.015 & Determination & 0.017 & Work Rate & 0.081 \\
First Touch & 0.009 & Concentration & 0.073 & Decisions & 0.014 \\
Reflexes & 0.079 & Leadership & 0.070 & Concentration & 0.033 \\
Throwing & 0.092 & Anticipation & 0.093 & Anticipation & 0.086 \\
Tendency to Punch & 0.030 & Positioning & 0.110 & Flair & 0.042 \\
Bravery & 0.011 & Composure & 0.074 & Positioning & 0.038 \\
Decisions & 0.083 & Teamwork & 0.045 & Teamwork & 0.017 \\
Concentration & 0.122 & Agility & 0.081 & Off the Ball & 0.047 \\
Anticipation & 0.119 & Stamina & 0.048 & Stamina & 0.029 \\
Positioning & 0.106 & Balance & 0.010 & Balance & 0.067 \\
Composure & 0.027 & Strength & 0.024 & Pace & 0.055 \\
Agility & 0.048 & Acceleration & 0.021 & Acceleration & 0.060 \\
Jumping & 0.054 & Jumping & 0.050 & Natural Fitness \\
\hline Inconsistency & 0.020 & Inconsistency & 0.030 & Inconsistency & 0.040 \\
\hline
\end{tabular}

Table 10: Priority weights for midfielder centres, wings, and forward centres

\begin{tabular}{llllll}
\hline Midfielder Centres & & Wings & & Forward Centres & \\
\hline Criteria & Priority & Criteria & Priority & Criteria & Priority \\
\hline Dribbling & 0.037 & Finishing & 0.016 & Finishing & 0.110 \\
First Touch & 0.011 & Dribbling & 0.073 & Dribbling & 0.010 \\
Marking & 0.019 & First Touch & 0.028 & First Touch & 0.052 \\
Passing & 0.044 & Crossing & 0.050 & Heading & 0.052 \\
Technique & 0.073 & Passing & 0.042 & Passing & 0.014 \\
Long Shots & 0.061 & Technique & 0.057 & Technique & 0.076 \\
Aggression & 0.009 & Long Shots & 0.022 & Long Shots & 0.014 \\
Bravery & 0.025 & Work Rate & 0.023 & Work Rate & 0.040 \\
Decisions & 0.031 & Decisions & 0.012 & Decisions & 0.058 \\
Determination & 0.030 & Determination & 0.011 & Determination & 0.020 \\
Leadership & 0.019 & Anticipation & 0.056 & Anticipation & 0.094 \\
Anticipation & 0.054 & Flair & 0.076 & Flair & 0.096 \\
Flair & 0.098 & Teamwork & 0.034 & Composure & 0.026 \\
Positioning & 0.049 & Off the Ball & 0.110 & Off the Ball & 0.095 \\
Composure & 0.053 & Agility & 0.099 & Agility & 0.045 \\
Teamwork & 0.128 & Stamina & 0.068 & Stamina & 0.018 \\
Off the Ball & 0.046 & Balance & 0.031 & Balance & 0.072 \\
Stamina & 0.077 & Pace & 0.087 & Pace & 0.028 \\
Strength & 0.095 & Acceleration & 0.062 & Acceleration & 0.047 \\
Acceleration & 0.041 & Natural Fitness & 0.043 & Jumping & 0.033 \\
\hline Inconsistency & 0.020 & Inconsistency & 0.010 & Inconsistency & 0.030 \\
\hline
\end{tabular}

\section{Parameters}

$V_{\text {ic }} \quad$ Score of criterion $c \in C$ of player $j \in P$

$\mathrm{W}_{\mathrm{rc}}$ Priority of criterion $\mathrm{c} \in \mathrm{C}$ of position $\mathrm{i} \in \mathrm{R}$

$A_{j} \quad$ Age of player $\mathrm{j} \in \mathrm{P}$

$\mathrm{C}_{\mathrm{j}} \quad$ Weekly cost of player $\mathrm{j} \in \mathrm{P}$

$\mathrm{Ps}_{\mathrm{j}} \quad$ Penalty kicking score of player $\mathrm{j} \in \mathrm{P}$

$\mathrm{Fs}_{\mathrm{j}} \quad$ Free kick score of player $\mathrm{j} \in \mathrm{P}$

$\mathrm{N}_{\mathrm{i}} \quad$ Maximum number of players who play in position $\mathrm{i} \in \mathrm{R}$

$\mathrm{U}$ Upper limit of average age of the first eleven

B Upper limit of weekly budget for team up

P Lower limit of penalty kicking score of the first eleven

F Lower limit of free kick score of the first eleven

Q Upper limit of number of foreign players in the first eleven 
Using the notation, the mathematical model is presented below, starting with the objective function.

Objective function

$$
\mathrm{Z}=\text { Maximise } \sum_{\mathrm{j}}^{\mathrm{P}} \sum_{\mathrm{i}}^{\mathrm{R}} \sum_{\mathrm{c}}^{\mathrm{C}} \mathrm{X}_{\mathrm{ji}} \mathrm{V}_{\mathrm{jc}} \mathrm{W}_{\mathrm{ic}}
$$

Subject To

$$
\begin{aligned}
& \sum_{\mathrm{i}}^{\mathrm{R}} \mathrm{X}_{\mathrm{ji}} \leq 1 \quad \forall_{\mathrm{j} \in \mathrm{P}} \\
& \sum_{\mathrm{j}}^{\mathrm{G}} \mathrm{X}_{\mathrm{ji}} \leq \mathrm{N}_{\mathrm{i}=1} \\
& \sum_{\mathrm{j}}^{\mathrm{D}} \mathrm{X}_{\mathrm{ji}} \leq \mathrm{N}_{\mathrm{i}=2} \\
& \sum_{\mathrm{j}}^{\mathrm{B}} \mathrm{X}_{\mathrm{ji}} \leq \mathrm{N}_{\mathrm{i}=3} \\
& \sum_{\mathrm{j}}^{\mathrm{M}} \mathrm{X}_{\mathrm{ji}} \leq \mathrm{N}_{\mathrm{i}=4} \\
& \sum_{\mathrm{j}}^{\mathrm{W}} \mathrm{X}_{\mathrm{ji}} \leq \mathrm{N}_{\mathrm{i}=5} \\
& \sum_{\mathrm{j}}^{\mathrm{F}} \mathrm{X}_{\mathrm{ji}} \leq \mathrm{N}_{\mathrm{i}=6} \\
& \sum_{\mathrm{j}}^{\mathrm{P}} \sum_{\mathrm{i}}^{\mathrm{R}} \mathrm{X}_{\mathrm{ji}} \mathrm{A}_{\mathrm{j}} \leq 11 \mathrm{U} \\
& \sum_{\mathrm{j}}^{\mathrm{P}} \sum_{\mathrm{i}}^{\mathrm{R}} \mathrm{X}_{\mathrm{ji}} \mathrm{C}_{\mathrm{j}} \leq \mathrm{B} \\
& \sum_{\mathrm{j}}^{\mathrm{P}} \sum_{\mathrm{i}}^{\mathrm{R}} \mathrm{X}_{\mathrm{ji}} \mathrm{Ps} \mathrm{s}_{\mathrm{j}} \geq \mathrm{P} \\
& \sum_{\mathrm{j}}^{\mathrm{P}} \sum_{\mathrm{i}}^{\mathrm{R}} \mathrm{X}_{\mathrm{ji}} \mathrm{Fs}_{\mathrm{j}} \geq \mathrm{F} \\
& \sum_{\mathrm{j}}^{\mathrm{S}} \mathrm{X}_{\mathrm{ji}} \leq \mathrm{Q} \\
& \mathrm{X}_{\mathrm{ji}} \in\{0,1\} \quad \forall_{\mathrm{j} \in \mathrm{P}, \mathrm{i} \in \mathrm{R}}
\end{aligned}
$$

The objective function minimises the total value of the players' talent. Constraint 2 ensures that each player must be assigned to only one position if the player is chosen. Constraints 3-8 provide that 11 players are selected for the team in line with the maximum available players for each position. Constraint 9 guarantees that the average age of the team must be lower than the upper limit of the average age. Constraint 10 ensures that the weekly cost of the team cannot exceed the club budget. Constraints 11 and 12 guarantee that the penalty and free kick value of the team must be equal to or greater than the lower limit respectively. Constraint 13 ensures that the number of assigned foreign players is equal to or lowers than the upper limit. Constraint 14 represents the binary variables.

\section{ILLUSTRATIVE CASE STUDY}

In this section, we present a case study to illustrate the effectiveness and applicability of the proposed approach.

\subsection{Description of the data}

The proposed approach is illustrated with real data obtained from the Fenerbahçe Soccer Club (FSC), a professional soccer team based in Istanbul, Turkey. Fenerbahçe has been in the first division of Turkish soccer since it was founded in 1905, and has never been relegated to lower divisions. The team squad has 30 players who include four goalkeepers, seven defensive centre players, seven fullbacks, twelve midfielder centre players, ten wings, and four forward centre players. Table 11 shows the players with their available positions and other data; as shown in this table, a player can play one or more positions. According to Table 11, Bruno Alves plays as a defensive centre player only, while Mehmet Topuz plays three different positions: fullback, midfielder centre, and wing.

As mentioned earlier, there are twenty criteria for each position. Tables $12-15$ give the scores of each criterion for six different player positions. It is noted that 20 is the maximum score for each criterion. All data was obtained from the Football Manager 2015 computer game. Bracketed numbers in Table 12 indicate the same players given in Table $11 . \mathrm{N}_{1,2,3,4,5,6}$ are set to 1, 2, 2, 2, 2, and 2 . The upper limit of the average age is set to 30 , and the weekly budget is US\$750,000. The lower limits of penalties and free kicks are 10. 
Table 11: Player list and related data

\begin{tabular}{|c|c|c|c|c|c|c|c|}
\hline No & Players & Available Positions & Cost in US\$ & Nation & Age & Penalty & $\begin{array}{l}\text { Free } \\
\text { Kick }\end{array}$ \\
\hline 1 & Volkan Demirel & Goalkeeper & 75000 & Local & 32 & 3 & 10 \\
\hline 2 & Mert Gunok & Goalkeeper & 16000 & Local & 25 & 1 & 10 \\
\hline 3 & Erten Ersu & Goalkeeper & 1100 & Local & 20 & 1 & 7 \\
\hline 4 & Ertugrul Taskiran & Goalkeeper & 2812 & Local & 24 & 3 & 10 \\
\hline 5 & Michal Kadlec & Fullback, Defensive Centre & 63000 & Foreign & 29 & 14 & 14 \\
\hline 6 & Hasan Ali Kaldırım & Fullback & 36000 & Local & 24 & 8 & 4 \\
\hline 7 & Caner Erkin & Fullback, Wing & 43000 & Local & 25 & 12 & 15 \\
\hline 8 & Serdar Kesimal & Fullback, Defensive Centre & 36000 & Local & 25 & 11 & 5 \\
\hline 9 & Bekir Irtegun & Fullback, Defensive Centre & 43500 & Local & 30 & 7 & 4 \\
\hline 10 & Gokhan Gonul & Fullback, Wing & 51000 & Local & 29 & 10 & 4 \\
\hline 11 & Mehmet Topuz & Fullback, Midfielder Centre, Wing & 40000 & Local & 30 & 15 & 13 \\
\hline 12 & Egemen Korkmaz & Defensive Centre & 53000 & Local & 31 & 10 & 6 \\
\hline 13 & Bruno Alves & Defensive Centre & 75000 & Foreign & 32 & 12 & 14 \\
\hline \multirow[t]{2}{*}{14} & & Defensive Centre, Midfielder & & Local & & & \\
\hline & Mehmet Topal & Centre & 62000 & & 28 & 10 & 7 \\
\hline \multirow[t]{2}{*}{15} & & Defensive Centre, Midfielder & & Local & & & \\
\hline & Selcuk Sahin & Centre & 28500 & & 33 & 8 & 5 \\
\hline 16 & Raul Meireles & Midfielder Centre & 75000 & Foreign & 31 & 12 & 14 \\
\hline 17 & Emre Belozoglu & Midfielder Centre & 60000 & Local & 33 & 16 & 14 \\
\hline 18 & Samuel Holmen & Midfielder Centre & 39000 & Foreign & 30 & 7 & 8 \\
\hline 19 & Gokay Irevul & Midfielder Centre & 2312 & Local & 21 & 8 & 12 \\
\hline 20 & Alper Potuk & Midfielder Centre, Wing & 45000 & Local & 23 & 10 & 10 \\
\hline 21 & Salih Ucan & Midfielder Centre & 4125 & Local & 20 & 12 & 9 \\
\hline 22 & Uygar Zeybek & Midfielder Centre, Wing & 1100 & Local & 19 & 5 & 6 \\
\hline 23 & Recep Niyaz & Midfielder Centre & 1100 & Local & 19 & 10 & 11 \\
\hline 24 & Diego Ribas & Midfielder Centre, Wing & 105000 & Foreign & 29 & 13 & 15 \\
\hline 25 & Miroslav Stoch & Wing & 54000 & Foreign & 24 & 12 & 13 \\
\hline 26 & Milos Krasic & Wing & 69000 & Foreign & 29 & 12 & 12 \\
\hline 27 & Dirk Kuyt & Wing, Forward Centre & 86000 & Foreign & 33 & 13 & 5 \\
\hline 28 & Moussa Sow & Wing, Forward Centre & 42000 & Foreign & 28 & 15 & 9 \\
\hline \multirow{3}{*}{$\begin{array}{l}29 \\
30\end{array}$} & Pierre Webo & Forward Centre & 37500 & Foreign & 32 & 14 & 10 \\
\hline & Emanuel & & & Foreign & & & \\
\hline & Emenike & Forward Centre & 72000 & & 27 & 12 & 7 \\
\hline
\end{tabular}

Table 12: Attribute values of goalkeepers and fullbacks

\begin{tabular}{lrrrr}
\hline & \multicolumn{4}{c}{ Goalkeepers } \\
\cline { 2 - 5 } Criteria & {$[1$} & {$[2$} & {$[3$} & {$[4$} \\
& {[} & ] & ] & ] \\
\hline Aerial Ability & 14 & 14 & 12 & 12 \\
Agility & 13 & 14 & 10 & 12 \\
Anticipation & 16 & 13 & 7 & 12 \\
Bravery & & & & \\
Command of Area & 16 & 17 & 13 & 13 \\
Communication & 10 & 12 & 12 & 12 \\
Composure & 14 & 13 & 7 & 12 \\
Concentration & 9 & 14 & 8 & 12 \\
Decisions & 15 & 12 & 12 & 11 \\
Eccentricity & 13 & 9 & 7 & 6 \\
First Touch & 9 & 9 & 2 & 8 \\
Handling & & & & \\
Jumping & 17 & 15 & 12 & 13 \\
Kicking & 15 & 16 & 14 & 14 \\
One on Ones & 17 & 13 & 12 & 17 \\
Positioning & 12 & 13 & 10 & 7 \\
Reflexes & 17 & 14 & 12 & 12 \\
Rushing Out & 15 & 15 & 12 & 14 \\
Tendency & 16 & 12 & 14 & 11 \\
Punch & & & & \\
Throwing & 10 & 10 & 6 & 13 \\
\hline & 14 & 12 & 11 & 16 \\
\hline
\end{tabular}

\begin{tabular}{|c|c|c|c|c|c|c|c|}
\hline \multirow[b]{2}{*}{ Criteria } & \multirow{3}{*}{5} & & & & & \multicolumn{2}{|c|}{ Fullbacks } \\
\hline & & \multirow[t]{2}{*}{6} & \multirow{2}{*}{$\begin{array}{c}7 \\
]\end{array}$} & \multirow{2}{*}{$\begin{array}{r}{[8} \\
]\end{array}$} & \multirow{2}{*}{$\begin{array}{l}{[9} \\
]\end{array}$} & \multirow{2}{*}{$\begin{array}{r}{[10} \\
]\end{array}$} & \multirow{2}{*}{ [11 } \\
\hline & & & & & & & \\
\hline Acceleration & 11 & 16 & 15 & 12 & 13 & 15 & 10 \\
\hline Anticipation & 14 & 10 & 12 & 12 & 13 & 14 & 11 \\
\hline $\begin{array}{l}\text { Balance } \\
\text { Concentratio }\end{array}$ & 14 & 9 & 13 & 12 & 13 & 11 & 15 \\
\hline $\mathrm{n}$ & 12 & 11 & 14 & 9 & 10 & 14 & 12 \\
\hline Corners & 12 & 7 & 16 & 2 & 5 & 7 & 11 \\
\hline Crossing & 15 & 10 & 17 & 8 & 7 & 12 & 15 \\
\hline Decisions & 13 & 10 & 9 & 11 & 11 & 14 & 9 \\
\hline Dribbling & 8 & 9 & 14 & 7 & 10 & 12 & 12 \\
\hline Flair & 8 & 8 & 14 & 8 & 5 & 14 & 9 \\
\hline Long Throws & 11 & 13 & 6 & 10 & 10 & 15 & 10 \\
\hline Marking & 13 & 11 & 9 & 12 & 12 & 13 & 12 \\
\hline Natural & & & & & & & \\
\hline Fitness & 15 & 16 & 15 & 10 & 12 & 18 & 15 \\
\hline Off the Ball & 10 & 10 & 12 & 11 & 7 & 12 & 9 \\
\hline Pace & 14 & 14 & 15 & 12 & 13 & 15 & 10 \\
\hline Positioning & 15 & 12 & 11 & 12 & 12 & 12 & 9 \\
\hline Stamina & 15 & 16 & 16 & 11 & 13 & 15 & 11 \\
\hline Tackling & 13 & 14 & 12 & 12 & 16 & 16 & 12 \\
\hline Teamwork & 16 & 13 & 12 & 11 & 12 & 15 & 15 \\
\hline Technique & & & & & & & \\
\hline & 10 & 12 & 14 & 12 & 8 & 13 & 13 \\
\hline Work Rate & 15 & 16 & 17 & 10 & 14 & 16 & 15 \\
\hline
\end{tabular}


Table 13: Attribute values of defensive centre and forward centre players

\begin{tabular}{|c|c|c|c|c|c|c|c|c|c|c|c|c|}
\hline \multirow[b]{2}{*}{ Criteria } & \multirow[b]{2}{*}{ [5] } & \multirow[b]{2}{*}{ [8] } & \multirow[b]{2}{*}{ [9] } & \multirow[b]{2}{*}[12]{} & \multicolumn{3}{|c|}{ Defence Players } & \multirow{2}{*}{ iteria } & \multicolumn{4}{|c|}{ Forward Players } \\
\hline & & & & & [13] & [14] & {$[15]$} & & [27] & [28] & [29] & {$[30]$} \\
\hline Acceleration & 11 & 12 & 13 & 10 & 9 & 11 & 11 & Acceleration & 10 & 16 & 12 & 16 \\
\hline Aggression & 12 & 11 & 11 & 17 & 18 & 13 & 14 & Agility & 14 & 16 & 14 & 16 \\
\hline Agility & 10 & 12 & 14 & 8 & 9 & 12 & 9 & Anticipation & 16 & 16 & 14 & 12 \\
\hline Anticipation & 14 & 12 & 13 & 13 & 16 & 16 & 13 & Balance & 16 & 16 & 15 & 18 \\
\hline Balance & 14 & 12 & 13 & 16 & 14 & 14 & 13 & Composure & 13 & 16 & 12 & 13 \\
\hline Bravery & 14 & 12 & 15 & 20 & 20 & 12 & 14 & Decisions & 15 & 13 & 13 & 9 \\
\hline Composure & 12 & 14 & 11 & 10 & 13 & 13 & 13 & Determination & 18 & 16 & 15 & 13 \\
\hline Concentration & 12 & 9 & 10 & 16 & 14 & 14 & 13 & Dribbling & 10 & 10 & 7 & 13 \\
\hline Decisions & 13 & 11 & 11 & 13 & 12 & 14 & 12 & Finishing & 12 & 18 & 14 & 16 \\
\hline Determination & 11 & 11 & 13 & 18 & 18 & 12 & 15 & First Touch & 15 & 12 & 15 & 13 \\
\hline Heading & 13 & 13 & 13 & 16 & 16 & 11 & 14 & Flair & 10 & 14 & 10 & 15 \\
\hline Jumping & 13 & 12 & 13 & 13 & 16 & 14 & 14 & Heading & 14 & 14 & 17 & 8 \\
\hline Leadership & 10 & 7 & 11 & 15 & 14 & 5 & 12 & Jumping & 12 & 11 & 13 & 13 \\
\hline Marking & 13 & 12 & 12 & 17 & 14 & 13 & 15 & Long Shots & 6 & 12 & 8 & 15 \\
\hline Passing & 13 & 13 & 9 & 8 & 13 & 13 & 12 & Off the Ball & 16 & 15 & 15 & 14 \\
\hline Positioning & 15 & 12 & 12 & 13 & 8 & 17 & 16 & Pace & 10 & 15 & 12 & 18 \\
\hline Stamina & 15 & 11 & 13 & 12 & 13 & 16 & 13 & Passing & 10 & 10 & 11 & 8 \\
\hline Strength & 13 & 12 & 13 & 17 & 18 & 14 & 13 & Stamina & 16 & 14 & 13 & 15 \\
\hline Tackling & 13 & 12 & 16 & 14 & 13 & 16 & 15 & Technique & 12 & 12 & 12 & 14 \\
\hline Teamwork & 16 & 11 & 12 & 13 & 14 & 18 & 16 & Work Rate & 19 & 15 & 15 & 14 \\
\hline
\end{tabular}

Table 14: Attribute values of midfielder centre players

\begin{tabular}{|c|c|c|c|c|c|c|c|c|c|c|c|c|}
\hline \multirow{2}{*}{ Criteria } & \multicolumn{12}{|c|}{ Players } \\
\hline & [11] & [14] & [15] & [16] & [17] & [18] & [19] & [20] & [21] & [22] & [23] & [24] \\
\hline Acceleration & 10 & 11 & 11 & 13 & 8 & 12 & 13 & 16 & 7 & 12 & 13 & 12 \\
\hline Aggression & 15 & 13 & 14 & 12 & 17 & 16 & 11 & 13 & 14 & 11 & 5 & 12 \\
\hline Anticipation & 11 & 16 & 13 & 15 & 17 & 14 & 11 & 11 & 13 & 12 & 11 & 14 \\
\hline Bravery & 18 & 12 & 14 & 10 & 16 & 15 & 8 & 12 & 5 & 7 & 7 & 9 \\
\hline Composure & 9 & 13 & 13 & 13 & 17 & 13 & 11 & 12 & 16 & 8 & 12 & 12 \\
\hline Decisions & 9 & 14 & 12 & 14 & 17 & 13 & 10 & 12 & 14 & 7 & 11 & 11 \\
\hline Determination & 16 & 12 & 15 & 13 & 19 & 15 & 13 & 14 & 11 & 13 & 13 & 11 \\
\hline Dribbling & 12 & 9 & 6 & 8 & 11 & 10 & 12 & 16 & 13 & 12 & 14 & 18 \\
\hline First Touch & 13 & 13 & 11 & 14 & 16 & 12 & 13 & 13 & 15 & 11 & 14 & 19 \\
\hline Flair & 9 & 8 & 6 & 11 & 16 & 10 & 11 & 14 & 15 & 13 & 17 & 18 \\
\hline Leadership & 9 & 5 & 12 & 12 & 16 & 11 & 7 & 8 & 7 & 10 & 8 & 5 \\
\hline Long Shots & 14 & 13 & 12 & 15 & 13 & 10 & 8 & 8 & 12 & 11 & 9 & 15 \\
\hline Marking & 12 & 13 & 15 & 13 & 10 & 12 & 8 & 9 & 6 & 9 & 3 & 6 \\
\hline Off the Ball & 9 & 8 & 11 & 12 & 12 & 14 & 9 & 12 & 11 & 8 & 11 & 11 \\
\hline Passing & 13 & 13 & 12 & 14 & 17 & 11 & 14 & 13 & 13 & 12 & 12 & 17 \\
\hline Positioning & 9 & 17 & 16 & 16 & 12 & 13 & 9 & 9 & 6 & 3 & 7 & 7 \\
\hline Stamina & 11 & 16 & 13 & 12 & 10 & 15 & 12 & 15 & 10 & 9 & 6 & 14 \\
\hline Strength & 14 & 14 & 13 & 12 & 12 & 10 & 6 & 8 & 7 & 6 & 5 & 9 \\
\hline Teamwork & 15 & 18 & 16 & 14 & 14 & 18 & 13 & 13 & 13 & 10 & 11 & 13 \\
\hline Technique & 13 & 12 & 11 & 14 & 17 & 11 & 14 & 15 & 18 & 13 & 16 & 19 \\
\hline
\end{tabular}

Table 15: Attribute values of wings

\begin{tabular}{|c|c|c|c|c|c|c|c|c|c|c|}
\hline \multirow{2}{*}{ Criteria } & \multicolumn{10}{|c|}{ Players } \\
\hline & [7] & [10] & [11] & [20] & [22] & [24] & [25] & [26] & [27] & [28] \\
\hline Acceleration & 15 & 15 & 10 & 16 & 12 & 12 & 17 & 14 & 10 & 16 \\
\hline Agility & 15 & 15 & 10 & 16 & 12 & 17 & 18 & 14 & 14 & 16 \\
\hline Anticipation & 12 & 14 & 11 & 11 & 12 & 14 & 7 & 13 & 16 & 16 \\
\hline Balance & 13 & 11 & 15 & 9 & 6 & 10 & 7 & 8 & 16 & 16 \\
\hline Crossing & 17 & 12 & 15 & 13 & 13 & 14 & 13 & 13 & 10 & 4 \\
\hline Decisions & 9 & 14 & 9 & 12 & 7 & 11 & 9 & 8 & 15 & 13 \\
\hline Determination & 17 & 17 & 16 & 14 & 13 & 11 & 7 & 14 & 18 & 16 \\
\hline Dribbling & 14 & 12 & 12 & 16 & 12 & 18 & 16 & 14 & 10 & 10 \\
\hline Finishing & 13 & 8 & 11 & 8 & 9 & 16 & 16 & 10 & 12 & 18 \\
\hline First Touch & 14 & 12 & 13 & 13 & 11 & 19 & 14 & 13 & 15 & 12 \\
\hline Flair & 14 & 14 & 9 & 14 & 13 & 18 & 15 & 11 & 10 & 14 \\
\hline Long Shots & 13 & 8 & 14 & 8 & 11 & 15 & 16 & 10 & 6 & 12 \\
\hline Natural Fitness & 15 & 18 & 15 & 14 & 17 & 14 & 14 & 7 & 17 & 12 \\
\hline Off the Ball & 12 & 12 & 9 & 12 & 8 & 11 & 11 & 15 & 16 & 15 \\
\hline Pace & 15 & 15 & 10 & 14 & 11 & 12 & 14 & 13 & 10 & 15 \\
\hline Passing & 15 & 13 & 13 & 13 & 12 & 17 & 14 & 13 & 10 & 10 \\
\hline Stamina & 16 & 15 & 11 & 15 & 9 & 14 & 13 & 12 & 16 & 14 \\
\hline Teamwork & 12 & 15 & 15 & 13 & 10 & 13 & 10 & 10 & 17 & 14 \\
\hline Technique & 14 & 13 & 13 & 15 & 13 & 19 & 16 & 15 & 12 & 12 \\
\hline Work Rate & 17 & 16 & 15 & 16 & 13 & 13 & 9 & 10 & 19 & 15 \\
\hline
\end{tabular}




\subsection{Computational experiment}

The 0-1 integer linear programming formulation (1)-(14) of the sample network contains 44 variables and 43 constraints. All computational experiments are conducted on a notebook computer with Intel Core2 Duo $1.66 \mathrm{GHz}$ and $2 \mathrm{~GB}$ RAM. The computation time required to solve the model to optimality using LINDO 14.0 is no more than one CPU second for any of the instances solved. The eleven best players obtained from the results are selected and shown in Figure 3. The total talent value of the first eleven - in other words, the objective function value - is calculated to be 144.689.

As seen from Figure 3, all constraints are satisfied. Five foreign players are selected for the first eleven. The average age, penalty kicking score and free kick score are 29.27, 11.54, and 11.09 respectively. To make a comparison, the last 38 matches of the FSC were investigated. Table 16 shows the first 16 players who take time most on the field. According to Table 13, 8 players out of 11 players who take time most, and 11 players out of 16 players who take time most are in the optimal solution. Players in the optimal solution are highlighted in bold.

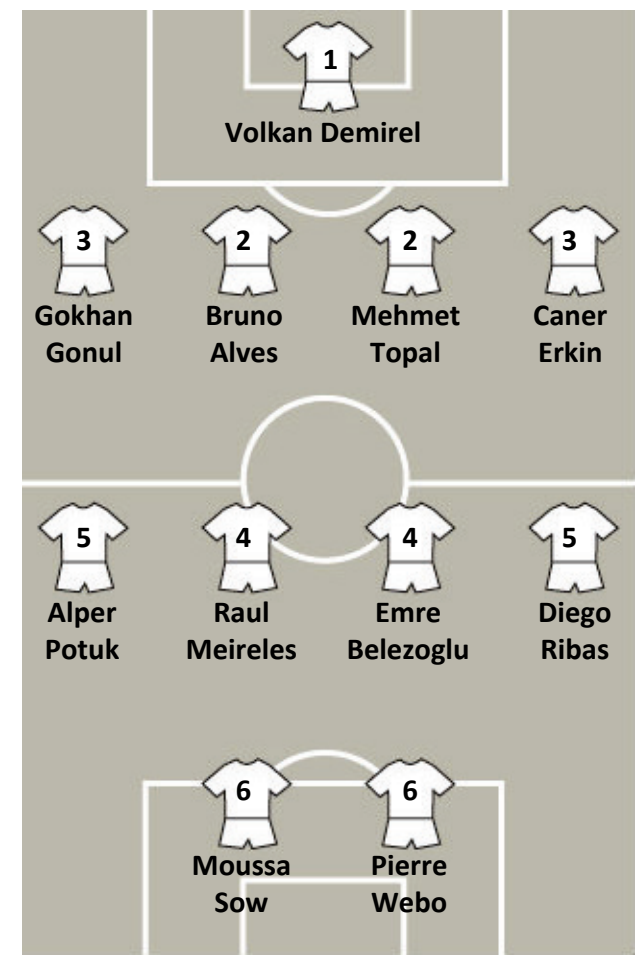

Figure 3: Optimal first eleven

Table 16: 16 players who take time most on the field [17]

\begin{tabular}{clrclr}
\hline Rank & Player & Total Time (min) & Rank & Player & Total Time (min) \\
\hline 1 & Mehmet Topal & 3321 & 9 & Bekir Irtegun & 2231 \\
2 & Gokhan Gonul & 2938 & 10 & Alper Potuk & 2080 \\
3 & Caner Erkin & 2790 & 11 & Emre Belozoglu & 1899 \\
4 & Moussa Sow & 2779 & 12 & Diego & 1742 \\
5 & Dirk Kuyt & 2547 & 13 & Michal Kadlec & 1615 \\
6 & Volkan Demirel & 2455 & 14 & Egemen Korkmaz & 1583 \\
7 & Bruno Alves & 2326 & 15 & Pierre Webo & 1576 \\
8 & Emmanuel Emenike & 2232 & 16 & Raul Meireles & 1515 \\
\hline
\end{tabular}

\subsection{Scenario analyses for managerial insights}

A number of instances were generated to carry out scenario analyses where problem parameters were changed to determine their impact on the performance measures. Each scenario is given below. It is noted that while changing some of the parameters, the rest of the parameters stay constant. 


\subsubsection{Scenario\# 1: Effects of the weekly budget}

The first scenario considered was the effect of decreasing the weekly budget of the club on the objective function and the team. The current budget was decreased five times with a 10 per cent decrement each time. Table 17 gives the selected players and objective function values of each run. According to Table 17, decreasing the weekly budget of the club decreases the total talent score of the team, and changes the team distribution, except for forward centre players.

Table 17: Effects of the weekly budget

\begin{tabular}{|c|c|c|c|c|c|c|}
\hline Positions & US\$ 375,000 & US\$ 450,000 & US\$ 525,000 & US\$ 600,000 & US\$ 675,000 & US\$ 750,000 \\
\hline Goalkeeper & $\begin{array}{l}\text { Ertugrul } \\
\text { Taskiran }\end{array}$ & Mert Gunok & Mert Gunok & Mert Gunok & $\begin{array}{l}\text { Volkan } \\
\text { Demirel }\end{array}$ & $\begin{array}{l}\text { Volkan } \\
\text { Demirel }\end{array}$ \\
\hline $\begin{array}{l}\text { Defensive } \\
\text { C.\#1 }\end{array}$ & Bruno Alves & Bruno Alves & Mehmet Topal & Bruno Alves & Bruno Alves & Bruno Alves \\
\hline $\begin{array}{l}\text { Defensive } \\
\text { C.\#2 }\end{array}$ & Selcuk Sahin & Selcuk Sahin & Selcuk Sahin & Selcuk Sahin & Mehmet Topal & Mehmet Topal \\
\hline Fullback\#1 & Caner Erkin & Caner Erkin & Caner Erkin & Caner Erkin & Caner Erkin & Caner Erkin \\
\hline Fullback\#2 & Mehmet Topuz & $\begin{array}{l}\text { Mehmet } \\
\text { Topuz }\end{array}$ & $\begin{array}{l}\text { Michael } \\
\text { Kadlec }\end{array}$ & Gokhan Gonul & Gokhan Gonul & Gokhan Gonul \\
\hline $\begin{array}{l}\text { Midfielder } \\
\text { C.\#1 }\end{array}$ & Salih Ucan & Salih Ucan & Salih Ucan & Raul Meireles & Raul Meireles & Raul Meireles \\
\hline $\begin{array}{l}\text { Midfielder } \\
\text { C.\#2 }\end{array}$ & Gokay Iravul & $\begin{array}{l}\text { Emre } \\
\text { Belozoglu }\end{array}$ & $\begin{array}{l}\text { Emre } \\
\text { Belozoglu }\end{array}$ & $\begin{array}{l}\text { Emre } \\
\text { Belozoglu }\end{array}$ & $\begin{array}{l}\text { Emre } \\
\text { Belozoglu }\end{array}$ & $\begin{array}{l}\text { Emre } \\
\text { Belozoglu }\end{array}$ \\
\hline Wing\#1 & Miroslav Stoch & Miroslav Stoch & Miroslav Stoch & Alper Potuk & Alper Potuk & Alper Potuk \\
\hline Wing\#2 & Alper Potuk & Alper Potuk & Diego Ribas & Diego Ribas & Diego Ribas & Diego Ribas \\
\hline Forward C.\#1 & Moussa Sow & Moussa Sow & Moussa Sow & Moussa Sow & Moussa Sow & Moussa Sow \\
\hline Forward C.\#2 & Pierre Webo & Pierre Webo & Pierre Webo & Pierre Webo & Pierre Webo & Pierre Webo \\
\hline Obj. Function & 135.197 & 139.502 & 141.628 & 143.568 & 144.689 & 144.689 \\
\hline
\end{tabular}

\subsubsection{Scenario\#2: Effects of the average age}

The other scenario considered was the effect of decreasing the average age of the team on the objective function and the team distribution. The current desired average age was decreased five times, one by one. Table 18 gives the selected players and objective function values of each run. According to Table 18, decreasing the average age of team also decreases the total talent score of the team, as found in the first scenario. The main reason for this is that the talent scores of young players are not as high as those of older players; it is understood that experience is very important for soccer players.

Table 18: Effects of the average age

\begin{tabular}{|c|c|c|c|c|c|c|}
\hline Positions & 25 years old & 26 years old & 27 years old & 28 years old & 29 years old & 30 years old \\
\hline Goalkeeper & Erten Ersu & Mert Gunok & Mert Gunok & Volkan Demirel & $\begin{array}{l}\text { Volkan } \\
\text { Demirel }\end{array}$ & $\begin{array}{l}\text { Volkan } \\
\text { Demirel }\end{array}$ \\
\hline $\begin{array}{l}\text { Defensive } \\
\text { C.\#1 }\end{array}$ & Michal Kadlec & Bruno Alves & Bruno Alves & Michael Kadlec & $\begin{array}{l}\text { Michael } \\
\text { Kadlec }\end{array}$ & Bruno Alves \\
\hline $\begin{array}{l}\text { Defensive } \\
\text { C.\#2 }\end{array}$ & Mehmet Topal & & Mehmet Topal & Bruno Alves & $\begin{array}{l}\text { Mehmet } \\
\text { Topal }\end{array}$ & $\begin{array}{l}\text { Mehmet } \\
\text { Topal }\end{array}$ \\
\hline Fullback\#1 & Mehmet Topuz & Michael Kadlec & Michael Kadlec & Caner Erkin & Caner Erkin & Caner Erkin \\
\hline Fullback\#2 & Caner Erkin & Caner Erkin & Caner Erkin & Gokhan Gonul & Gokhan Gonul & Gokhan Gonul \\
\hline $\begin{array}{l}\text { Midfielder } \\
\text { C.\#1 }\end{array}$ & Gokay Irevul & Salih Ucan & Salih Ucan & Salih Ucan & Raul Meireles & Raul Meireles \\
\hline $\begin{array}{l}\text { Midfielder } \\
\text { C.\#2 }\end{array}$ & Recep Niyaz & Recep Niyaz & Emre Belozoglu & Emre Belozoglu & $\begin{array}{l}\text { Emre } \\
\text { Belozoglu }\end{array}$ & $\begin{array}{l}\text { Emre } \\
\text { Belozoglu }\end{array}$ \\
\hline Wing\# 1 & Alper Potuk & Alper Potuk & Alper Potuk & Alper Potuk & Alper Potuk & Alper Potuk \\
\hline Wing\#2 & Miroslav Stoch & Diego Ribas & Miroslav Stoch & Diego Ribas & Diego Ribas & Diego Ribas \\
\hline Forward C.\#1 & Moussa Sow & Moussa Sow & Moussa Sow & Moussa Sow & Moussa Sow & Moussa Sow \\
\hline Forward C.\#2 & $\begin{array}{l}\text { Emanuel } \\
\text { Emenike }\end{array}$ & $\begin{array}{l}\text { Emanuel } \\
\text { Emenike }\end{array}$ & $\begin{array}{l}\text { Emanuel } \\
\text { Emenike }\end{array}$ & $\begin{array}{l}\text { Emanuel } \\
\text { Emenike }\end{array}$ & Pierre Webo & Pierre Webo \\
\hline Obj. Function & 132.321 & 138.517 & 141.017 & 143.447 & 144.332 & 144.689 \\
\hline
\end{tabular}

\section{CONCLUSION}

In this paper, a two-phase solution approach was proposed for soccer player selection. In the first phase, the attributes of each player based on each position were prioritised using AHP. In the second phase, a 0-1 integer linear programming model was developed using the weights of player attributes, and the top performers were determined for inclusion in the team. The proposed solution approach was applied to the Turkish soccer club Fenerbahçe, in order to illustrate the applicability and performance of the approach. 
The contribution of the proposed method is twofold: (1) it fills the gaps in the literature with regard to quantitative applications that enable effective and efficient player selection and team formation; (2) it applies a hybrid approach that combines AHP and 0-1 integer programming model to select soccer players for a particular team. It should be accepted that the proposed approach does not imply a higher-level of 'accuracy' in player selection and team formation. However, the authors believe that the proposed approach may help coaches to think systematically about player selection problems and to improve the quality of their decisions. It is noted that the proposed approach is not only applicable to soccer player selection: it can also be used in other multi-player sports. One suggestion for future research is to embed imprecise or ambiguous judgments into the decisionmaking process. Finally, a decision support system that uses the proposed solution approach should be developed for general use.

\section{ACKNOWLEDGMENT}

The authors express sincere appreciation to the editor and anonymous reviewers for their efforts to improve the quality of this paper. Author was supported by the BAGEP Award of the Science Academy in Turkey.

\section{REFERENCES}

[1] Tavana, M., Azizi, F., Azizi, F. and Behzadian, M. 2013. A fuzzy inference system with application to player selection and team formation in multi-player sports. Sport Management Review, 16(1), pp. 97-110.

[2] Sarda, V., Sakaria, P. and Deulkar, K. 2015. Football team selection using genetic algorithm. International Journal of Engineering and Technical Research, 3(2), pp. 153-156.

[3] Ahmed, F., Deb, K. and Jindal, A. 2013. Multi-objective optimization and decision making approaches to cricket team selection. Applied Soft Computing, 13(1), pp. 402-414.

[4] Boon, B.H. and Sierksma, G. 2003. Team formation: Matching quality supply and quality demand. European Journal of Operational Research, 148(2), pp. 277-292.

[5] Sharp, G.D., Brettenny, W.J., Gonsalves, J.W., Lourens, M. and Stretch, R.A. 2011. Integer optimization for the selection of a Twenty-20 cricket team. Journal of the Operational Research Society, 62, pp. 16881694.

[6] Merigo, J.M. and Gil-Lafuente, A.M. 2011. Decision-making in sport management based on the OWA operator. Expert Systems with Applications, 38(8), pp. 10408-10413.

[7] Kamble, A.G., Rao, R.V., Kale, A. and Samant, S. 2011. Selection of cricket players using analytical hierarchy process. International Journal of Sports Science and Engineering, 5(4), pp. 207-212.

[8] Omkar, S.N. and Verma, R. 2003. Cricket team selection using genetic algorithm. International Congress on Sports Dynamics, Melbourne, Australia, pp. 1-9.

[9] Gerber, H. and Sharp, G.D. 2006. Selecting a limited overs cricket squad using an integer programming model. South African Journal for Research in Sport, Physical Education and Recreation, 28, pp. 81-90.

[10] Amin, G.R. and Sharma, S.K. 2014. Cricket team selection using data envelopment analysis. European Journal of Sport Science, 14(1), pp. 369-376.

[11] http://www.fm-base.co.uk/forum/. Accessed on 18 February 2016.

[12] Saaty, T.L. 1980. The Analytic hierarchy process: Planning, priority setting and resource allocation. New York: McGraw-Hill.

[13] Huang, H.C., Lin, C.T. and Hu, C.S. 2011. Analysis of selection indicators of badminton players by the Delphi method and analytic hierarchy process. International Journal of Computer Science \& Information Technology, 7(1), pp. 19-31.

[14] Saaty, T.L. 2008. Decision making with the analytic hierarchy process. International Journal of Services Sciences, 1(1), pp. 83-98.

[15] Bruno, G., Esposito, E., Genovese, A. and Passaro, R. 2012. AHP-based approaches for supplier evaluation: Problems and perspectives. Journal of Purchasing and Supply Management, 18(3), pp. 159-172.

[16] Erkan, T.E. and Can, G.F. 2014. Selecting the best warehouse data collecting system by using AHP and FAHP methods. Technical Gazette, 21(1), pp. 87-93.

[17] http://www.mackolik.com/Takim/2/Fenerbahce\#. Accessed on 18 February 2016. 